\title{
Saturn's Atmospheric Temperature Structure and Heat Budget
}

\author{
GLENN S. ORTON \\ Earth and Space Sciences Division, Jet Propulsion Laboratory, California Institute of Technology \\ Pasadena, California 91103
}

ANDREW P. INGERSOLL

Division of Geological and Planetary Sciences, California Institute of Technology

Pasadena, California 91125

\begin{abstract}
The effective temperature of Saturn from $30^{\circ} \mathrm{S}$ to $10^{\circ} \mathrm{N}$ is $96.5 \pm 2.5 \mathrm{~K}$. This value is $1.9 \mathrm{~K}$ higher than our preliminary estimate (Ingersoll et al., 1980). The atmospheric mole fraction of $\mathrm{H}_{2}$ relative to $\mathrm{H}_{2}+\mathrm{He}$ is $90 \pm 3 \%$. This value is derived by comparing infrared and radio occultation data (Kliore et al., this issue) for the same latitude. The high value of the effective temperature suggests that Saturn has an additional energy source besides cooling and contraction. The high mole fraction of $\mathrm{H}_{2}$ suggests that separation of heavier He toward the core may be supplying the additional energy. Atmospheric temperatures in the 60- to 600 -mbar range are $2.5 \mathrm{~K}$ lower within $7^{\circ}$ of the equator than at higher latitudes. An almost isothermal layer exists between 60 and 160 mbar at all latitudes.
\end{abstract}

\section{INTRODUCTION}

In our preliminary report [Ingersoll et al., 1980] of the Pioneer Saturn infrared radiometer (IRR) results we discussed the data set and viewing geometry and presented a preliminary analysis based on early postencounter trajectory information. We now present a more complete analysis. This paper discusses Saturn's infrared emissions at 20- and $45-\mu \mathrm{m}$ wavelength, the atmospheric thermal structure from $30^{\circ} \mathrm{S}$ to $10^{\circ} \mathrm{N}$ latitude and from 60- to 600-mbar pressure, the global energy budget of Saturn, and the atmospheric $\mathrm{H}_{2}$ to He ratio. Another paper [Froidevaux and Ingersoll, this issue] discusses Saturn's rings and Titan.

Some of the numbers have changed since our preliminary report. The changes are all within the error limits quoted in the preliminary report, but they are nevertheless interesting. First, the estimated effective temperature of Saturn is higher by $1.9 \mathrm{~K}$, a change attributable to a different treatment of long-wave emission beyond the range of instrument sensitivity. This change, plus the likely possibility that Saturn scatters a higher proportion of incident sunlight into large phase angles than Jupiter [Tomasko et al., this issue], raises the estimate of Saturn's internal energy flux significantly. The other major change is that our results have now been thoroughly integrated with those from the Pioneer radio occultation experiment [Kliore et al., this issue], and a new estimate of the hydrogen to helium ratio has been obtained. Our preliminary use of earth-based spectra to infer the stratospheric temperature gradient led to a high value for the hydrogen abundance [Kliore et al., 1980]. Now, by using the radio occultation results to constrain the stratospheric temperature profile the mole fraction of hydrogen from the two experiments is $90 \pm$ $3 \%$. (The quoted error reflects uncertainties in the IRR data only.) These two changes are relevant to discussions of Saturn's internal history, of whether separation of hydrogen and helium is occurring, and of whether such separation is contributing to Saturn's internal energy output.

The next section gives the basic 20 - and $45-\mu \mathrm{m}$ data for Saturn. From these data and a knowledge of the filter and cali-

Copyright (C) 1980 by the American Geophysical Union. bration factors, which are also given, one can calculate all the results that follow. Then we describe the temperature-sounding method. The results - temperature profiles, latitudinal structure, whole disk infrared spectra, and net infrared heat budget-follow in succeeding sections. A final section discusses implications for global energy budget and interior models.

\section{BASIC DATA}

Observations of the planet cover a range of emission angle cosine $\mu$ from 1.0 to 0.2 . Unambiguous observations of emission at $\mu=0.2$ were possible because of the relatively close trajectory of the spacecraft to the planet, in terms of planetary radius. For comparison the lowest value of $\mu$ used for Jupiter was 0.4 .

As in the Jupiter observations, no point on Saturn was observed more than once. Therefore in order to acquire a useful set of intensity measurements in each channel which would be useful for atmospheric structure retrieval, longitudinal homogeneity of the atmosphere was assumed. For the latitudes over which data were acquired from both rising and setting sides of the central meridian (i.e., for latitudes where occultation by the ring system did not occur), no asymmetries between the intensities of the rising and setting limbs were observed above the variance of the data about the mean in either channel. Unlike the Jupiter data, latitude binning of the data was not done in preselected regions representing major morphological features, since relatively little was known about the properties of the planet as a function of latitude. Instead, adjacent bins were used, with equal widths of $3^{\circ}$ in latitude. This size allows a substantial number of data to be gathered for averaging over the widest possible area of the planet without unduly smoothing over the apparent extent of major bright and dark infrared regions, using the infrared map shown by Ingersoll et al. [1980, Figure 1] as a guide. For each channel the set of latitude-binned data was fit by a three-term polynomial

$$
I(\mu)=I_{0} P_{0}(\mu)+I_{1} P_{1}(\mu)+I_{2} P_{2}(\mu)
$$

This polynomial provided a good fit to the total data set without imposing superfluous variations in the limb structure. 


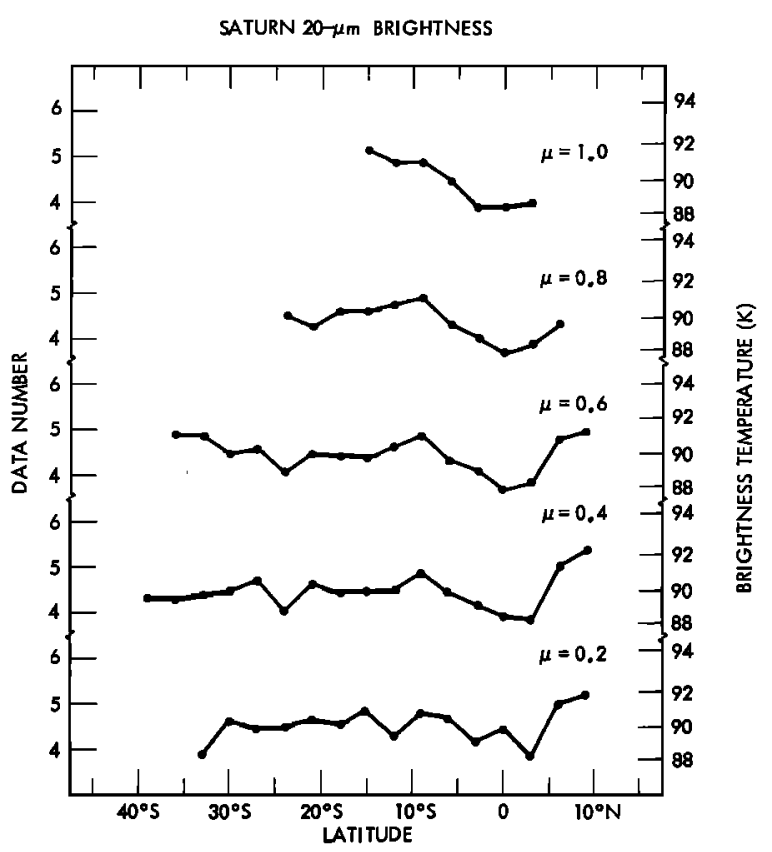

Fig. 1. Brightness of Saturn at 20- $\mu \mathrm{m}$ wavelength. Raw data numbers are shown at left, and brightness temperature scale at right. Five values of the emission angle cosine $\mu$ are shown.

Higher-order terms did not provide a better fit to the data.

Figures 1 and 2 show the basic data for the 20- and $45-\mu \mathrm{m}$ channels, respectively. For each latitude bin centered at $0^{\circ}$, $\pm 3^{\circ}, \pm 6^{\circ}$, etc., the coefficients $I_{0}, I_{1}$, and $I_{2}$ were determined along with the $3 \times 3$ error matrix computed from the scatter of the data about (1). From this information, values of $I(\mu)$ and their uncertainties were computed at $\mu=0.2,0.4, \cdots, 1.0$. Only values whose uncertainties are less than $\pm 0.5 \mathrm{DN}$ are plotted. (DN is the data number; the conversion to brightness temperature is shown in the scale at the right in the figures.) Restricting the plotted curves in this way eliminates latitude- $\mu$ combinations for which we have no data or for which a modest extrapolation in $\mu$ is not possible. For instance, we have observations at $\mu=1.0$ only at one point on the planet near $6^{\circ} \mathrm{S}$ latitude, but a modest extrapolation from $\mu=0.98$ enables us to plot the $\mu=1.0$ curves from $15^{\circ} \mathrm{S}$ to $3^{\circ} \mathrm{N}$.

Calibration procedures were described following the Jupiter encounters [Ingersoll et al., 1976] and in the preliminary Saturn report [Ingersoll et al., 1980]. The primary calibration was done in the laboratory before launch. Part of the prelaunch calibration involved measuring the response to an internal shutter at the known instrument temperature. After launch the instrument temperature and the response to the shutter were monitored. This response is measured with respect to liquid nitrogen in the laboratory and with respect to space during flight. The emission from liquid nitrogen requires a $3 \%$ correction at $45 \mu \mathrm{m}$ and a $0.2 \%$ correction at $20 \mu \mathrm{m}$. In accord with laboratory measurements the instrument response in space is represented by a linear relation. Values of intercept and slope are derived from observations of space and the shutter, respectively. These derivations were repeated at least once per year from 1973 to 1980 and during the encounters with Jupiter and Saturn as planetary data were being taken. The difference between the derived response to a $100-\mathrm{K}$ object and the laboratory value was no more than $3 \%$ for all these determinations. The instrument seems to have been stable to $\pm 3 \%$ for 8 years. Our total estimates of uncertainty in the absolute cali- bration fall in the range \pm 4 to $\pm 8 \%$. As with the Jupiter data, the latter value will be used throughout this report.

\section{TEMPERATURE-SOUNDING METHOD}

The determination of the atmospheric structure of Saturn with IRR measurements follows essentially the same techniques as the analysis of Pioneer 10 and 11 IRR data on Jupiter [Orton, 1975; Orton and Ingersoll, 1976]. We assume that the collision-induced dipole of $\mathrm{H}_{2}$, under the influence of both $\mathrm{H}_{2}$ and $\mathrm{He}$ collisions, provides most of the atmospheric opacity. While the opacity of $\mathrm{NH}_{3}$ was also included in the calculations, as in the Jovian case, the inclusion of its opacity has a negligible effect on the atmospheric transmission in the spectral regions covered by the two channels. This is due to the low amount of $\mathrm{NH}_{3}$, whose abundance is presumed to follow saturation equilibrium, in this relatively cold region of the atmosphere. The weighting functions are sensitive to somewhat lower pressures than are those for the Jovian atmosphere owing primarily to the greater value of the atmospheric scale height for Saturn. This larger scale height is the result of the lower gravitational acceleration of Saturn compared with that of Jupiter.

The vertical range and resolution for temperature sounding were evaluated from the weighting functions shown in Figure 3, using Conrath's [1972] adaptation of Backus-Gilbert theory. These estimates have been found [Orton, 1977] to be consistent with the temperature retrieval limitations of Chahine's [1975] technique, which is the method used in this work. The result is shown in Figure 4. It is apparent that meaningful retrieval of the temperature can be done only in the range 0.06 $\leq p \leq 0.50$ bar and that the vertical resolution averages about 0.3 in units of $\log _{10} p$ (bar), approximately 0.7 pressure scale heights. We have thus attempted to retrieve the temperature at four vertical levels (nodal points) where temperatures can be retrieved independently of one another, corresponding to $\log _{10} p$ (bar) $=-0.3,-0.6,-0.9$, and $-1.2(p=0.501,0.251$, 0.126 , and 0.063 bar, respectively). Between these levels, temperatures are interpolated linearly in $\log p$. We have restricted the range of latitudes chosen for temperature sounding so that for each channel, observations at each latitude were available for $0.7 \geq \mu \geq 0.3$. For such values of $\mu$ the vertical resolution and range are virtually unchanged from those shown in Figure 4 , which were computed for $1.0 \geq \mu \geq 0.2$.

In the deep atmosphere $\left(\log _{10} p \geq-0.3(p \geq 501 \mathrm{mbar})\right)$, we have assumed an adiabatic temperature lapse rate. For $\log _{10} p$

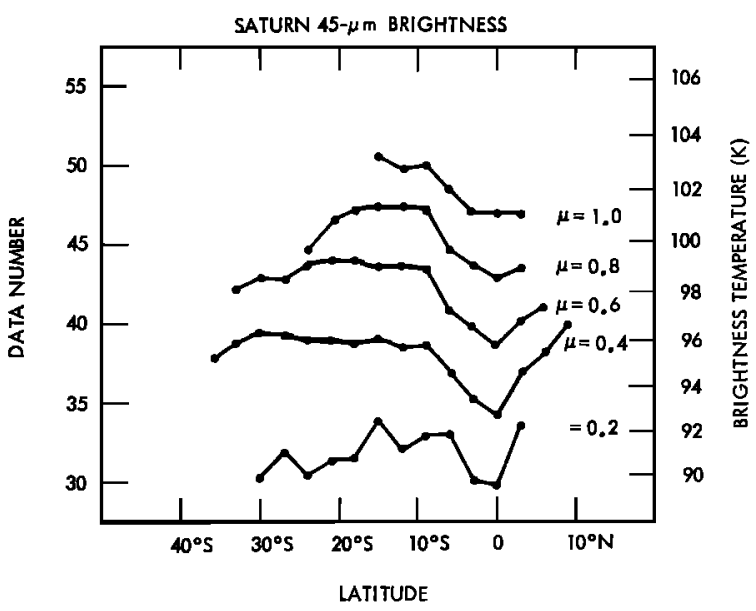

Fig. 2. Same as Figure 1 at $45-\mu \mathrm{m}$ wavelength. 
$\leq-1.2$ ( $p \leq 63$ mbar) we have, for the sake of completeness, assumed two alternative values for the inverted lapse rate. One corresponds to the mean lapse rate in the lower portion of the radio occultation profile [Kliore et al., this issue], approximately $21 \mathrm{~K}$ of increase per decade of pressure decrease; the other corresponds to the mean lapse rate in the lower portion of the inversion in models best matching earth-based observations of thermal emission from Saturn [Tokunaga and Cess, 1977], a steeper gradient at approximately $36 \mathrm{~K}$ of increase per decade of pressure decrease. The profiles of both Kliore et al. and Tokunaga and Cess are shown in Figure 12 and will be discussed later in more detail. These lapse rates are used to extrapolate the thermal structure down to $\log _{10} p$ (bar) $=2.0$ (100 bars) and up to $\log _{10} p$ (bar) $=-3.2(0.63$ mbar), where radiative transfer calculations were discontinued, since temperature changes below and above this level do not affect radiation observed in either channel. In the numerical calculation of the radiative transfer integral the atmosphere was divided into finite homogeneous layers, 10 per decade of pressure change. The temperature-sounding iterations were continued conservatively for $\mathbf{3 0}$ iterations, so that the changes in recovered temperatures at any given level between the next to last and the last iteration were usually well below $0.1 \mathrm{~K}$. Model test recoveries with synthetic data, to which the observed level of noise was added, show that the uncertainty in the temperatures recovered at a given nodal point from the technique itself (as opposed to systematic errors or uncertainties in the opacity models or in the calibration) is of the order of 3 or $4 \mathrm{~K}$, with a worst case of $6 \mathrm{~K}$ in the neighborhood of the temperature minimum. The root mean square residuals of a model fit to the data were computed by

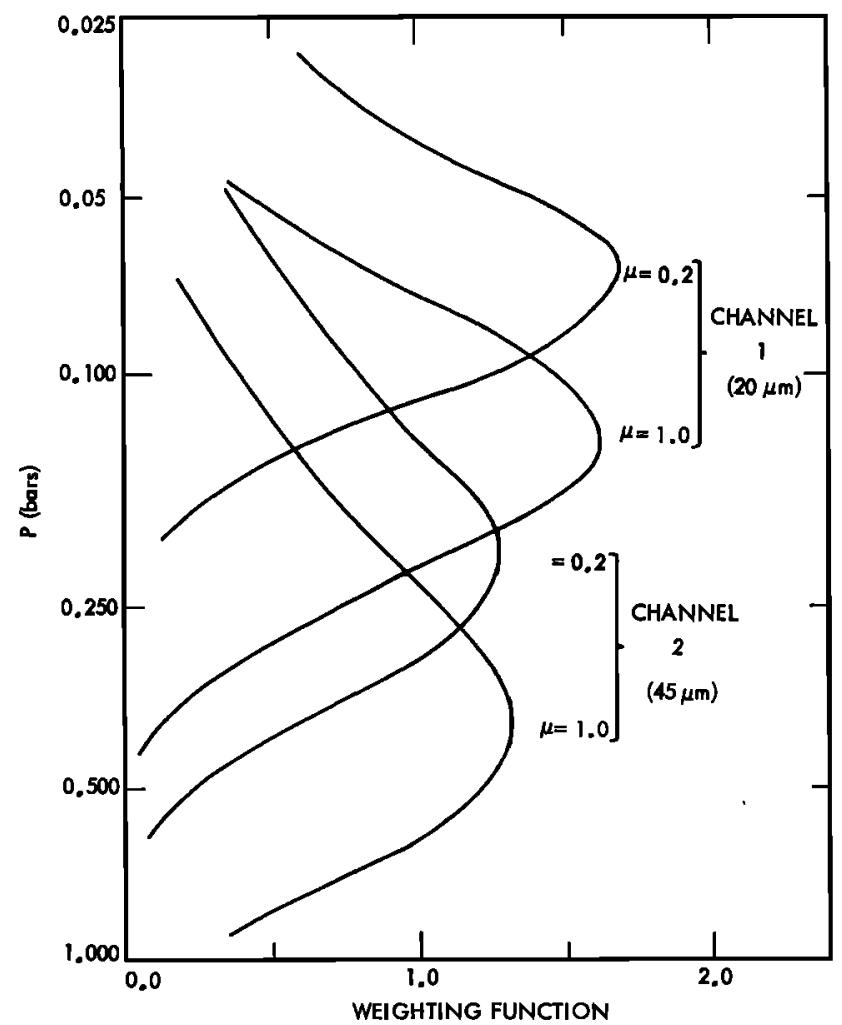

Fig. 3. Effective weighting functions for the infrared radiometer at Saturn. The mixing ratios of $\mathrm{H}_{2}$ and $\mathrm{He}$ are 0.90 and 0.10 , respectively, in this model. The weighting function is plotted in arbitrary units.

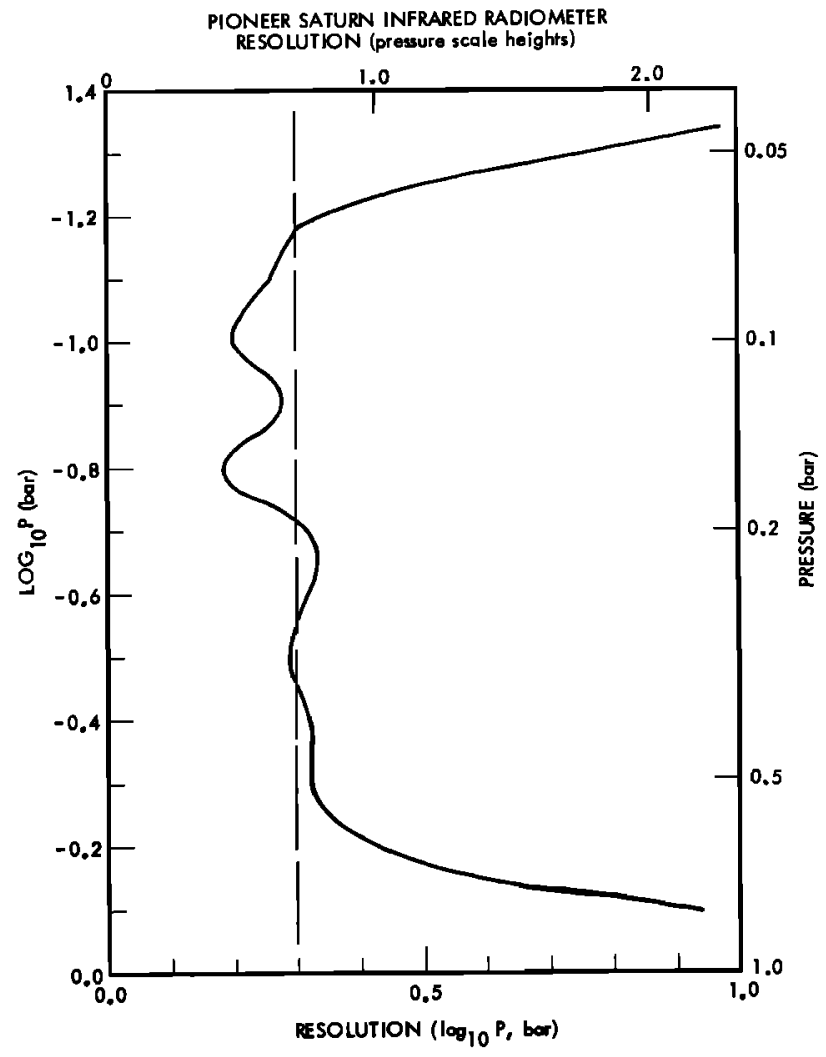

Fig. 4. Spread function, inverse of the vertical resolution, for temperature sounding with the IRR experiment. This was computed according to the method of Conrath [1972].

$$
\epsilon=\left[\sum_{i=1}^{N}\left(\left.\frac{T_{B}^{\text {model }}-T_{B}^{\text {data }}}{T_{B}^{\text {data }}}\right|^{2} /(N-1)\right]^{1 / 2}\right.
$$

where $i$ represents a (channel, $\mu$ ) pair, $T_{B}$ is a brightness temperature, and the sum is taken over data in both channels. Here the 'data' are intensities $I(\mu)$ computed from (1).

\section{COMPARISON WITH RADIO OCCULTATION RESULTS: BULK COMPOSITION}

The radio occultation experiment [Kliore et al., this issue] provides data down to $125 \mathrm{mbar}$, in the middle of the altitude range covered by the IRR. The exit data yield a profile of atmospheric refractivity at a latitude of $9.6^{\circ} \mathrm{S}$. This latitude was also sampled by the IRR, although at different longitudes, at times differing by at most a few hours. The profile of temperature versus pressure that one derives from the radio occultation data depends on the assumed composition, represented here by $\alpha_{H_{2}}$, the number of $H_{2}$ molecules relative to $H_{2}+\mathrm{He}$. Figure $12 b$ of Kliore et al. [this issue] gives a family of such profiles for various assumptions about the value of this parameter. The temperature profile that one derives from the IRR data also depends on $\alpha_{\mathrm{H}_{2}}$, but in a different way. Thus for any value of $\alpha_{\mathrm{H}_{2}}$ one can use the appropriate radio occultation profile to get the temperature above the 126-mbar level and allow the IRR data to determine temperatures at the two lowest nodal points at $\log _{10} p$ (bar) $=-0.3$ and $-0.6(0.501$ and 0.251 bar). The derived value of $\alpha_{\mathrm{H}_{2}}$ is the one that gives the lowest $\mathrm{rms}$ residual $\epsilon$, defined in (2).

Figure 5 displays the residuals for each temperature sounding as a function of the $\mathrm{H}_{2}$ mixing ratio assumed. They are minimized for $\alpha_{\mathrm{H}_{2}}=0.90 \pm 0.02\left(\alpha_{\mathrm{He}}=0.10 \pm 0.02\right)$. The un- 


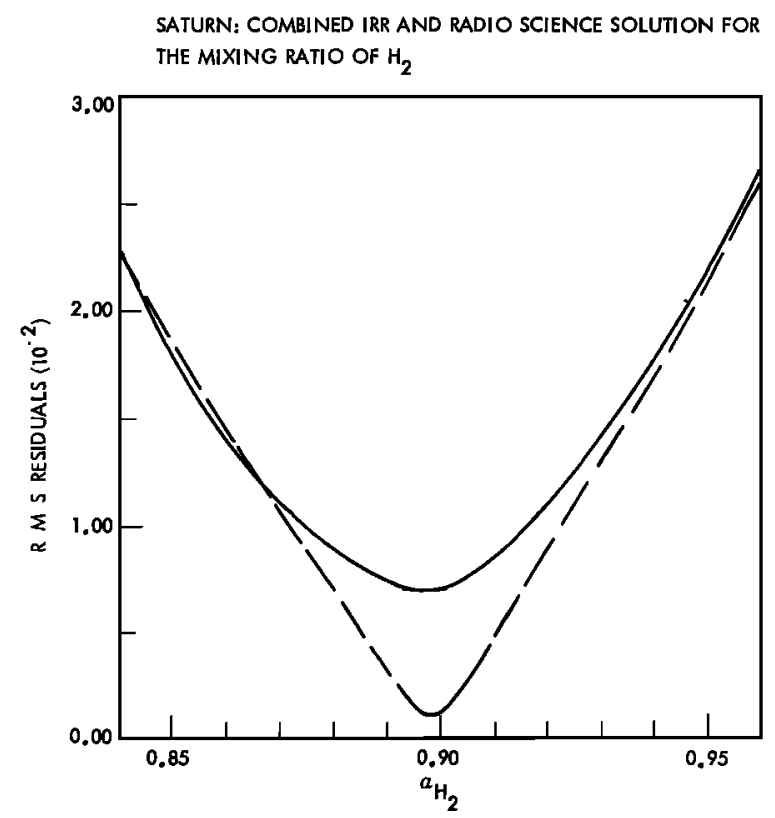

Fig. 5. Root mean square residuals to the fit between IRR data and outgoing fluxes computed from the radio occultation model as a function of the hydrogen mixing ratio $\alpha_{H_{3}}$. The mixing ratio of helium is assumed to be complementary. The solid curve represents the residuals to the unweighted fit to the data set; the dashed curve represents the residuals to the fit weighted as described in the text.

certainty quoted is derived from the equation used by Orton and Ingersoll [1976], conservatively assuming only 1 degree of freedom, and it represents only the uncertainty of the fit. The minimum residual value is $6.9 \times 10^{-3}$, of the same order as residuals for this latitude from straightforward temperature sounding, discussed in the next section, for the full 0.501 - to 0.063 -bar range. The resulting thermal structure is shown in Figure 6.

As a test of the method we tried weighting the residuals in each pair of (channel, $\mu$ ) sets according to its degree of overlap with the radio occultation results -strongly favoring the fit to residuals to the $20-\mu \mathrm{m}$ channel results near the limb (small values of $\mu$ ). These weighted residuals were minimized for $\alpha_{\mathbf{H}_{2}}$ $=0.90$. Since there is only one significant observation (intensity at $20 \mu \mathrm{m}$ for small $\mu$ ) and one free parameter $\left(\alpha_{H_{2}}\right)$, the number of degrees of freedom is zero, and an uncertainty estimate would be meaningless. Nevertheless, the result is consistent with that obtained by consideration of the full data set.

This comparison between radio occultation and IRR results is valid only under the assumption that the longitudes sounded by the radio occultation experiment are representative of the average temperature structure in this latitude region over all longitudes. Further implicit assumptions are required for either the radio occultation or the infrared remote retrieval of temperatures (using the IRR data set) to be valid in general: (1) the atmosphere is in thermodynamic equilibrium, (2) all constituents besides $\mathrm{H}_{2}$ and $\mathrm{He}$ are negligible at the level of error in the fit, (3) the atmosphere is spheroidally symmetric, with isobar surfaces completely coincident with geopotential surfaces, and an appropriate value for the planetary oblateness has been used, (4) the atmosphere is in hydrostatic equilibrium, (5) the infrared opacity is modeled correctly, (6) the thermal structure is longitudinally homogeneous, and (7) the absolute calibration of the radiances measured by the IRR experiment is accurate.

Violation of the above assumptions leads to additional uncertainties in $\alpha_{H_{2}}$. For example, an 8\% overestimate of the 20- $\mu \mathrm{m}$ intensity leads to a 1-K overestimate of temperature near the 0.06-bar level, corresponding to a 0.01 underestimate of $\alpha_{\mathrm{H}_{2}}$. Errors in opacity tend to have a smaller effect, because the atmosphere at these altitudes is nearly isothermal, so that the pressure at which the minimum temperature occurs is irrelevant. The addition of the few levels of radio occultation results for $p>125$ mbar makes an insignificant change in the results. The effect of clouds near the 500 -mbar level on outgoing radiation, as will be discussed below, has no effect on these results, as the region of overlapping coverage is substantially higher in the atmosphere. Furthermore, an examination of Figure 13 of Kliore et al. [this issue] shows that uncertainties in the retrieved radio occultation temperature structure due to the assumption of an initial temperature or to bias selection are negligible in the region of overlap with IRR results. The values of $90 \% \mathrm{H}_{2}$ and $10 \% \mathrm{He}$ by volume differ from the initial results given by Kliore et al. [1980], because their earlier comparison was made between IRR results derived by assuming a much steeper inverted lapse rate in the stratosphere than is consistent with the radio occultation results.

Temperature differences between the IRR range of longitudes and those sampled by the radio signal are more difficult to estimate. A 1-K difference in temperature would lead to a 0.01 change in $\alpha_{\mathrm{H}_{2}}$. Nonequilibrium, nonhydrostatic effects in the atmosphere are also difficult to estimate, as are systematic effects in the radio occultation experiment besides those discussed above. The temperature error in the overlap region due to radio data alone is thought to be less than $\pm 1 \mathrm{~K}$ (A. J. Kliore, personal communication, 1980).

Our final results, then, using this technique are

$$
\begin{aligned}
& \alpha_{H_{2}}=0.90 \pm 0.03 \\
& \alpha_{H_{e}}=0.10 \pm 0.03
\end{aligned}
$$

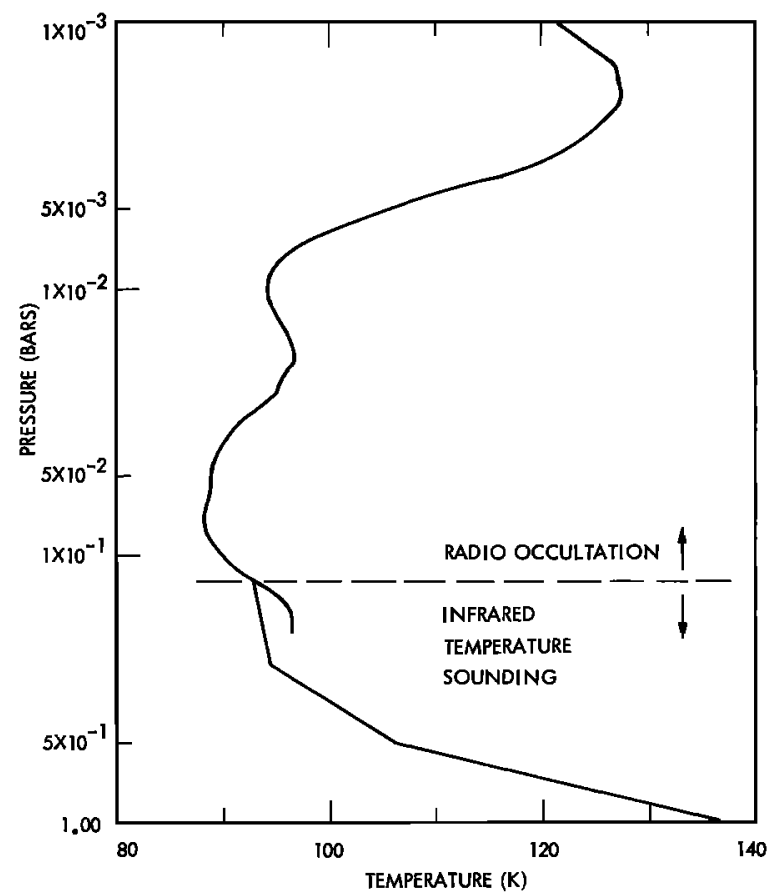

Fig. 6. Retrieved temperature structure for $8.1^{\circ}-11.1^{\circ} \mathrm{S}$ latitude, centered on the region sounded by the Pioneer 11 egress radio occultation [Kliore et al., this issue]. The radio occultation profile is used for $p \leq 126$ mbar. Residuals of the model fit to IRR data are minimized by (1) temperature sounding the region where $p \geq 126 \mathrm{mbar}$ and (2) adjusting the mixing ratios of $\mathrm{H}_{2}$ and $\mathrm{He}$ as shown in Figure 5 . 
with no uncertainties in the values quoted other than those corresponding to the internal fit and to the radiometer calibration. All other potential sources of systematic uncertainty following the assumptions listed above cannot be estimated in quantitative terms. These values are assumed in all temperature-sounding calculations discussed below.

We note that the method used by Orton and Ingersoll [1976] to determine the relative abundances of $\mathrm{H}_{2}$ and $\mathrm{He}$ in Jupiter took advantage of an overlap between the 20 - and the $45-\mu \mathrm{m}$ channel vertical coverage of the atmosphere. This extra degree of freedom allowed a test of a variety of values of the $\mathbf{H}_{2}$ and $\mathrm{He}$ mixing ratios to be made, since the collision-induced opacity of $\mathrm{H}_{2}$ in the $45-\mu \mathrm{m}$ channel versus the $20-\mu \mathrm{m}$ channel is somewhat sensitive to the relative number of $\mathrm{H}_{2}$ versus $\mathrm{He}$ collisions with the $\mathrm{H}_{2}$ molecule. As Figure 3 shows, however, this degree of freedom was absent for the Saturn atmosphere; that is, there was no overlap in the vertical coverage of the atmosphere between the two channels without making unrealistic assumptions about the integrity of the few 45- $\mu \mathrm{m}$ data collected near low-latitude regions characterized by values of $\mu \lesssim$ 0.10 .

The technique of correlating radio occultation and infrared results has been used for Jupiter in a comparison of Voyager 1 radio science system (RSS) and infrared interferometer spectrometer (IRIS) data. The derived value for the $\mathrm{H}_{2}$ mixing ratio is $\alpha_{\mathbf{H}_{2}}=0.897 \pm 0.024$ [Gautier et al., 1980]. This value is remarkably close to the value derived here for Saturn. A comparison of the Voyager 1 and 2 RSS and IRIS results should be made for Saturn also to determine the bulk composition, following the respective spacecraft encounters with that planet. These should produce results which do not require the assumption of longitudinal homogeneity of the atmospheric structure to the same extent as is required for IRR data analysis.

\section{TEMPERATURE-SOUNDING Results}

Temperature soundings were made at latitudes from $30^{\circ} \mathrm{S}$ to $9^{\circ} \mathrm{N}$ in bins $3^{\circ}$ wide. The radio occultation profile was not used to constrain the data at $p<125 \mathrm{mbar}$, as it was in the last section. Rather, the 20 - and $45-\mu \mathrm{m}$ data were used to solve for temperature at the four nodal points $\log _{10} p$ (bar) $=-0.3$, $-0.6,-0.9$, and $-1.2(p=0.501,0.251,0.126$, and $0.063 \mathrm{bar})$. Several assumptions were made regarding (1) the value of the inverted lapse rate (temperature increase with altitude) in the $\log _{10} p<-1.2$ region ( $p \leq 63$ mbar) and (2) the pressure of clouds in the troposphere. The three major cases discussed below are summarized in Table 1 . Recall that an overlying lapse rate of $21 \mathrm{~K}$ per decade of pressure is consistent with the radio occultation profile at $9.6^{\circ} \mathrm{S}$ (cases $l$ and 3). An overlying lapse rate of $36 \mathrm{~K}$ per decade of pressure is more consistent with earth-based observations (case 2). Crude cloud models, where invoked (case 3), consist of a unit emissivity surface representing a uniform, optically thick cloud top emitting at the ambient temperature.

TABLE 1. Temperature-Sounding Assumptions

\begin{tabular}{ccl}
\hline Case & $\begin{array}{c}\text { Overlaying } \\
\text { Lapse Rate, } \\
\text { K/log } p\end{array}$ & Cloud Presence \\
\hline 1 & 21 & no \\
2 & 36 & no \\
3 & 21 & where required \\
(see text)
\end{tabular}

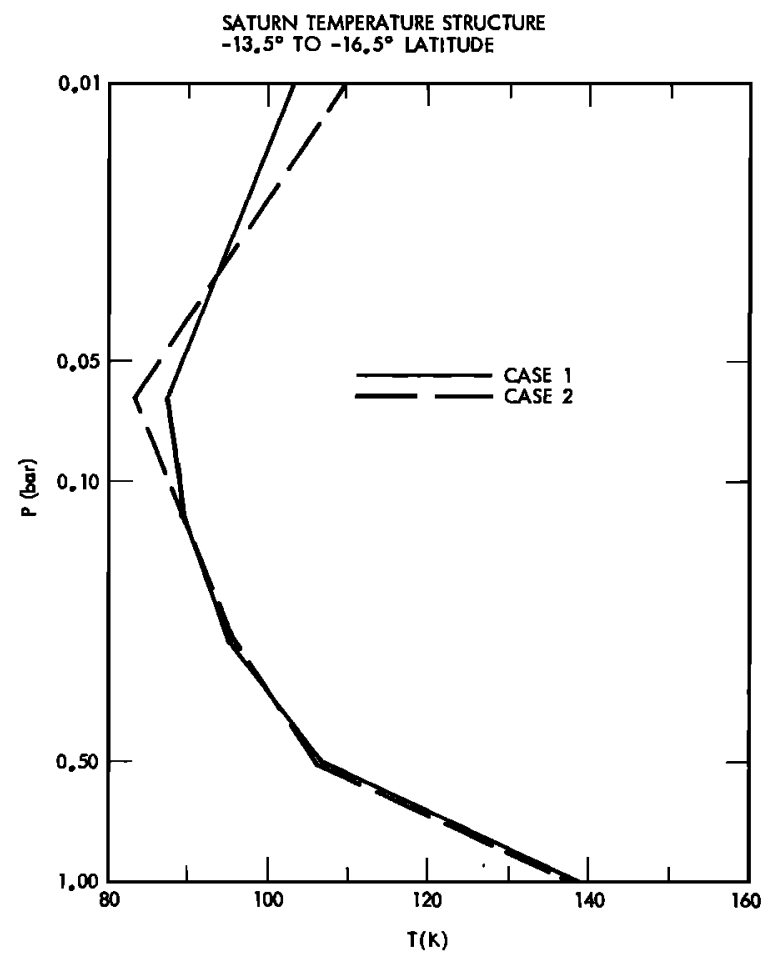

Fig. 7. Retrieved temperature structure for cases 1 and 2, described in the text and in Table 1 , for data from a latitude region extending from $13.5^{\circ} \mathrm{S}$ to $16.5^{\circ} \mathrm{S}$, one of the warmest regions examined.

Figures 7 and 8 display the vertical temperature structures retrieved from two latitude regions. They are (1) $13.5^{\circ}-$ $16.5^{\circ} \mathrm{S}$, a region which is one of the warmest observed and which is associated with a visually dark area in reflected sunlight (properties similar to a Jovian belt) and (2) $1.5^{\circ} \mathrm{N}$ to $1.5^{\circ} \mathrm{S}$, a region which is one of the coldest observed and which

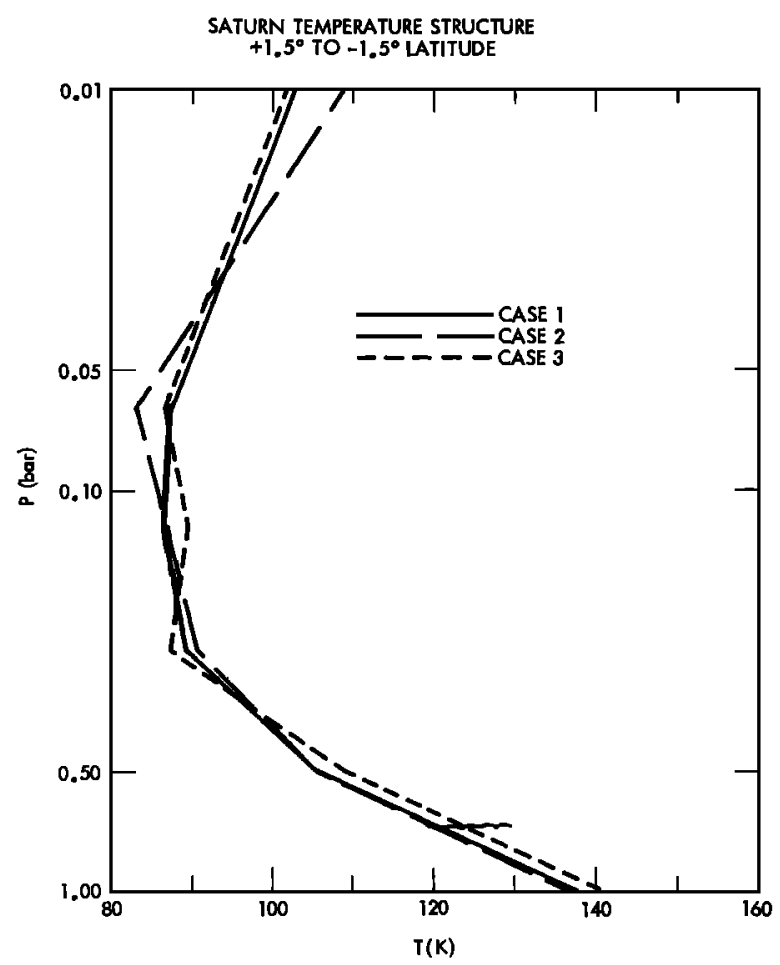

Fig. 8. Retrieved temperature structure for cases 1,2 , and 3 for data from a latitude region extending from $1.5^{\circ} \mathrm{N}$ to $1.5^{\circ} \mathrm{S}$, one of the coolest regions examined. The location of a cloud top, modeled as a unit emissivity blackbody surface, is also marked. 

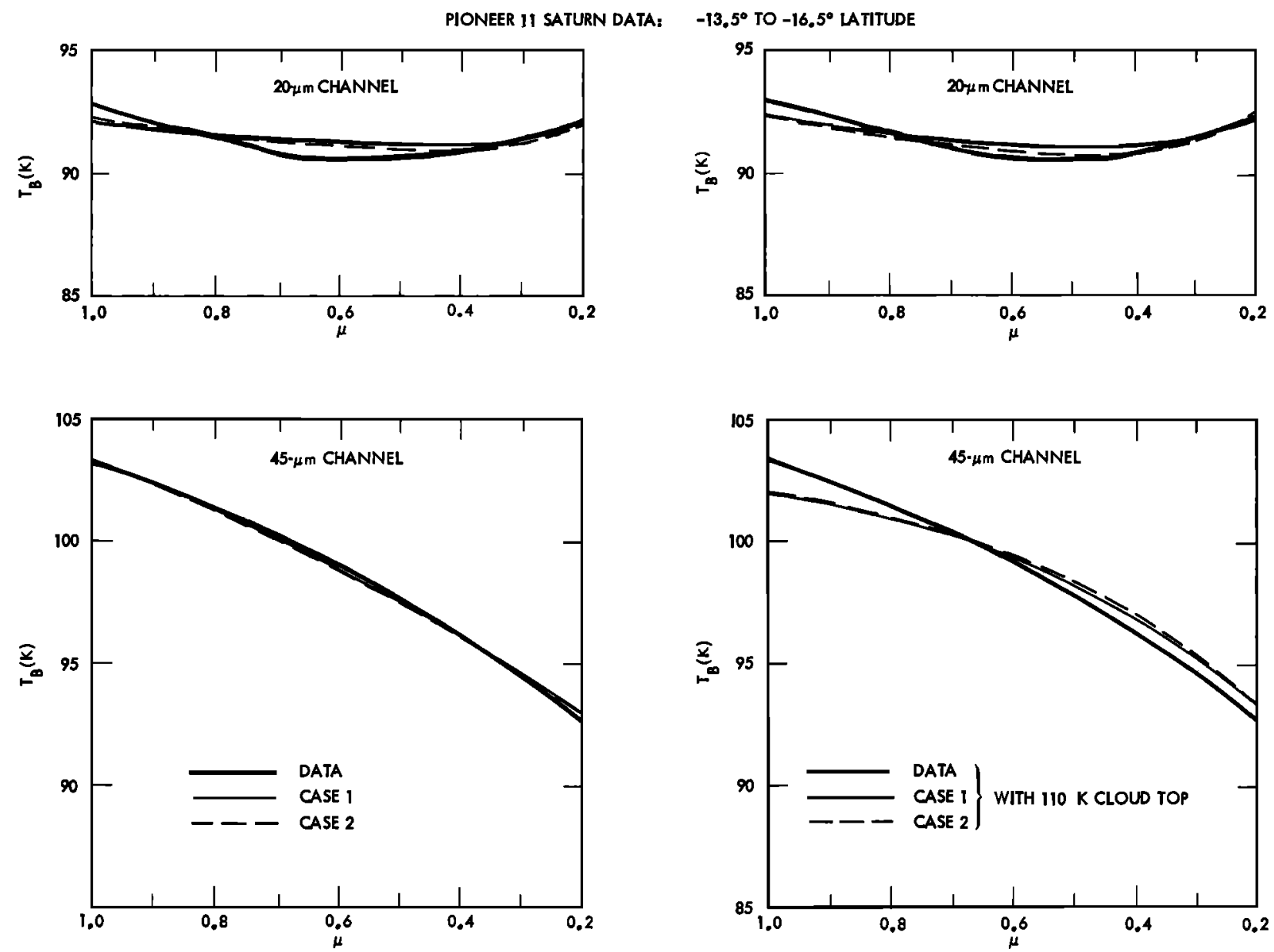

Fig. 9. Fit of various retrieved models to data from the relatively warm $13.5^{\circ}-16.5^{\circ} \mathrm{S}$ latitude region.

is associated with a visually bright area in reflected sunlight (properties similar to a Jovian zone), for Figures 7 and 8, respectively.

Clear atmospheres, free of the absorption or scattering effects of any aerosols, were assumed in cases 1 and 2. Figures 7 and 8 show the vertical temperature structure associated with each of these cases for the two latitude regions described above. The lower part of the retrieved profiles, between $\log _{10} p$ $=-0.6$ and -0.3 ( $501 \geq p \geq 251$ mbar), is characterized by lapse rates which are slightly subadiabatic and therefore not inconsistent with the assumption of adiabatic lapse rates at deeper levels. We note, however, that we cannot rule out the possibility of a subadiabatic lapse rate at levels deeper than 501 mbar, except by reference to a variety of radiative-convective equilibrium models, all of which predict convective equilibrium in this region (e.g., J. F. Appleby and J. Hogan, unpublished manuscript, 1980). A comparison between these theoretical models and the results derived here will be made later.

The difference between the overlying lapse rates assumed for cases 1 and 2 appears to affect only the temperatures recovered at the highest nodal point (63 mbar) to any substantial degree. In addition to the generally colder temperatures, compared with the Jovian temperature structure (e.g., 500 -mbar temperatures near $105 \mathrm{~K}$ instead of approximately $143 \mathrm{~K}$ for Jupiter), the next major difference between these temperature profiles and those derived for Jupiter is the rather shallow lapse rate in the vicinity of the temperature minimum. In fact, for case 1 the equatorial cool region is nearly isothermal for more than an atmospheric scale height (Figure 8).

In case 3 we pursued the possibility that the differences in the retrieved temperatures at 501 mbar between cases 1 and 2 result from changes in the properties of aerosols near that level in the atmosphere. This corresponds to the alternative model for temperatures near the 700-mbar level in Jovian zones. For this 'cloudy zone' model we assumed that the 501mbar level of the atmosphere is in convective equilibrium and that latitudinal variations of temperature along isobars are well below our observational noise. For the equatorial zones, then, we introduced a uniform, flat, and optically opaque cloud top with unit emissivity. Its altitude was adjusted until the retrieved temperature at 501 mbar was near $108 \mathrm{~K}$, an appropriate mean of temperatures retrieved at this depth in higher-latitude regions where aerosol effects were presumed to be negligible.

The vertical structure and cloud location for this case are shown in Figure 8 for the equatorial 'zone.' If the cloud is real and, further, if it is similar to the Jovian ammonia ice cloud, a consistent picture may be found in which the $\mathrm{NH}_{3}$ cloud or haze is substantially thicker (at least at some latitudes) than its Jovian counterpart. The effective blackbody cloud top temperatures for Jovian zones are near $148 \mathrm{~K}$ [Orton, 1975; Orton and Ingersoll, 1976], quite close to the saturation temperature for $\mathrm{NH}_{3}$. The Saturn cloud top temperatures in the 

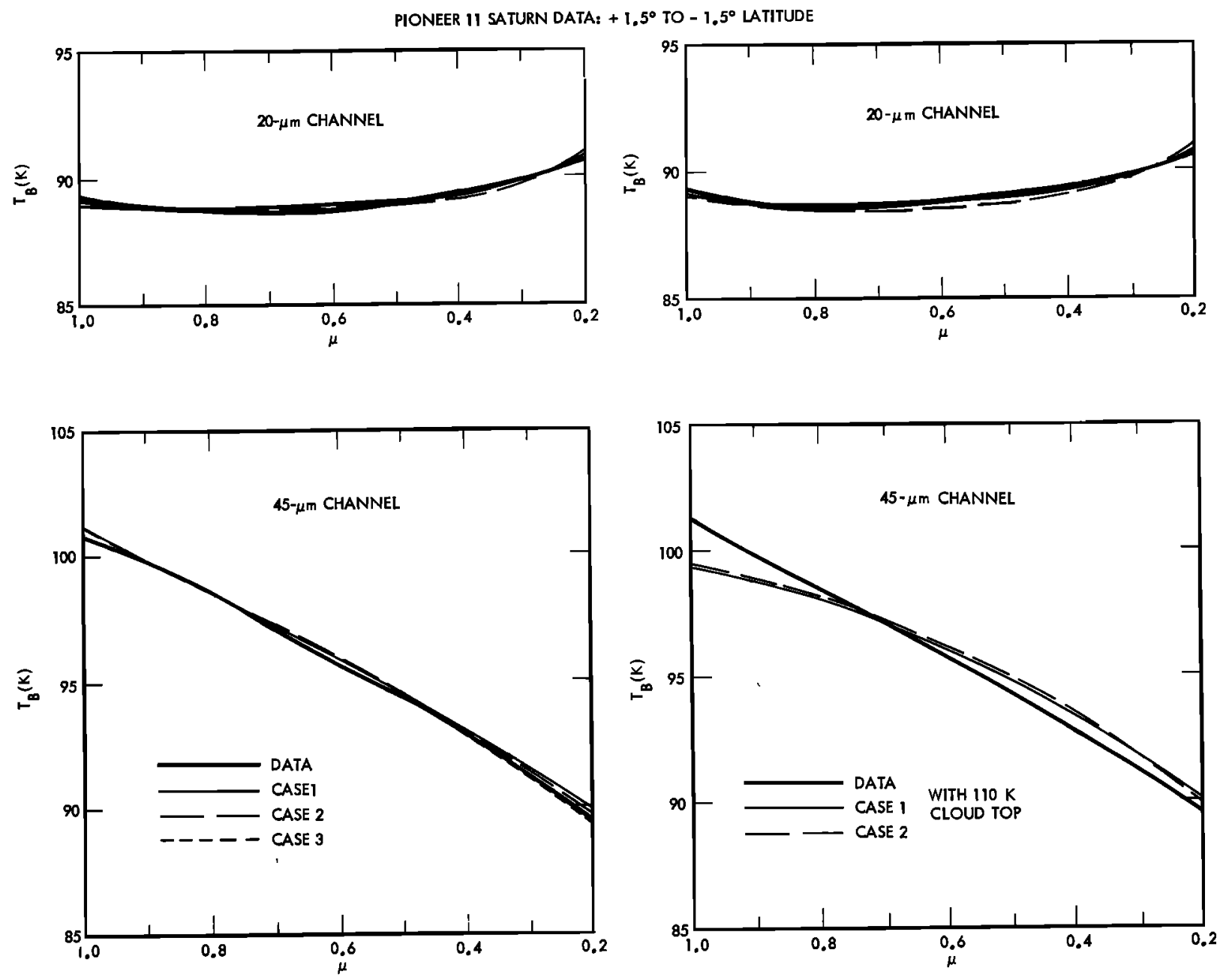

Fig. 10. Same as Figure 9 but for the relatively cool $1.5^{\circ} \mathrm{N}$ to $1.5^{\circ} \mathrm{S}$ latitude region.

$10^{\circ} \mathrm{N}$ to $10^{\circ} \mathrm{S}$ latitude region ( $124 \mathrm{~K}$ ) are far too low to be explained solely by saturation equilibrium (which would require a substantial enrichment of ammonia abundance in the deep atmosphere of Saturn relative to that of Jupiter). However, the ammonia saturation level, if it is near temperatures of 148 $\mathbf{K}$, is relatively deep within the convective region of Saturn. Thus it is plausible to consider that the aerosols may be carried aloft by convection to regions where the ambient temperatures are well below those at the saturation level.

Caldwell s [1977] analysis of the 8- to 14- $\mu \mathrm{m}$ spectrum of the Saturn disk, observed by Gillett and Forrest [1974], requires, in fact, an optically opaque cloud top temperature just below $110 \mathrm{~K}$. We pursued that model by assuming that a $110-\mathrm{K}$ optically opaque cloud exists in the temperature profile retrieval. However, such models give a consistently worse fit to the data than the models in cases 1,2 , and 3 . The fits of various model cases to the data are shown in Figures $9\left(13.5^{\circ}-16.5^{\circ} \mathrm{S}\right)$ and 10 $\left(1.5^{\circ} \mathrm{N}\right.$ to $\left.1.5^{\circ} \mathrm{S}\right)$. It is clear that cases 1,2 , and 3 provide good fits to the data in each respective region, with root mean square residuals (equation (2)) of the order of $3.9 \times 10^{-3}$ and $2.5 \times 10^{-3}$. For these same regions, models with an opaque cloud top at $110 \mathrm{~K}$ yield residuals of $6.7 \times 10^{-3}$ and $7.5 \times 10^{-3}$ because of the inability of the models to provide sufficient limb darkening to match the $45-\mu \mathrm{m}$ data, as Figures 9 and 10 show.
We conclude that these models do not represent vaiable alternatives. The difference between the optimum models for the $8-$ to $14-\mu \mathrm{m}$ region and the $45-\mu \mathrm{m}$ regions is that there is probably a substantial wavelength dependence of the optical properties of aerosols (presumably $\mathrm{NH}_{3}$ ice crystals). In order to be consistent with both the $10-\mu \mathrm{m}$ and the $45-\mu \mathrm{m}$ regions the cloud must be more opaque at $10 \mu \mathrm{m}$ that at $45 \mu \mathrm{m}$. An examination of Taylor's [1973] calculation of the extinction efficiency $Q_{\text {ext }}$ for $\mathrm{NH}_{3}$ ice shows that cloud particles would be more transparent at longer wavelengths if the particles were characterized by mode radii of the order of 1-10 $\mu \mathrm{m}$. Whether this is the case for ammonia ice in the atmosphere of Saturn must be determined by application of radiative transfer calculations which include scattering and absorption by $\mathrm{NH}_{3}$ ice crystals, which is outside the scope of this report.

A smoothed plot of temperatures between 60 - and 500mbar altitude and between $30^{\circ} \mathrm{S}$ and $10^{\circ} \mathrm{N}$ latitude is shown in Figure 11. The equatorial zone is a prominent cold region at all altitudes. But in comparison with Jupiter there are remarkably few features at other latitudes. The same behavior is seen in the raw data shown in Figures 1 and 2. We have searched for evidence of ring shadowing in these data. The Saturnocentric latitude of the sun was $2.83^{\circ} \mathrm{S}$ at the time of the encounter. Northern spring equinox occurred 51 days later. The only possible evidence of ring shadowing is the 


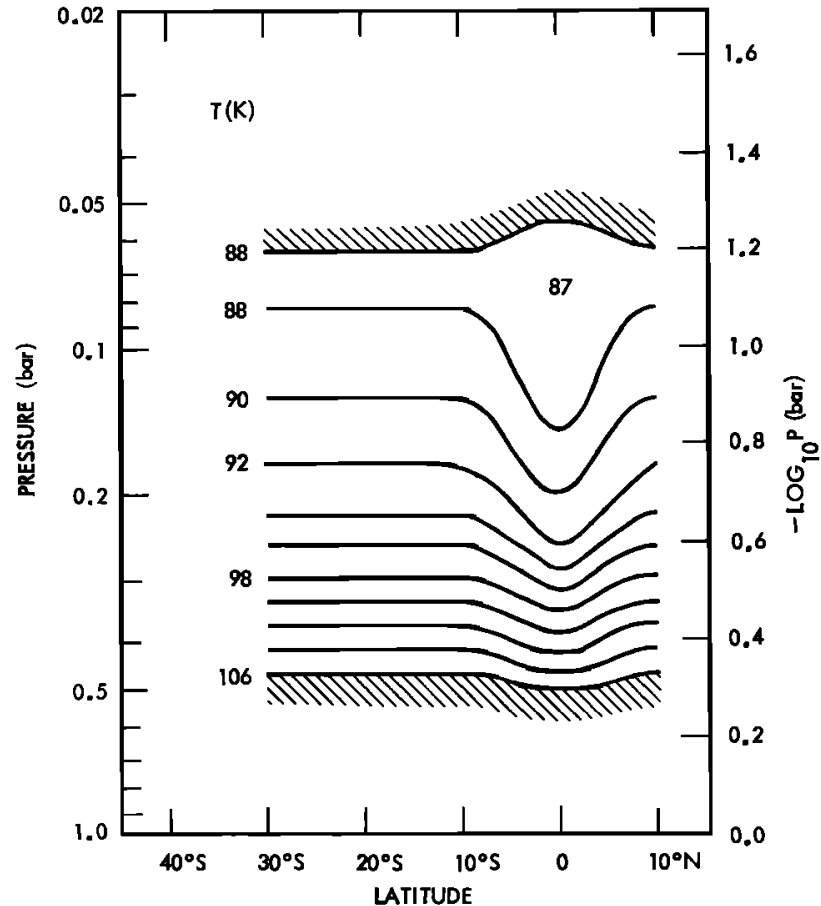

Fig. 11. Smooth contours of temperature in a meridional cross section from $30^{\circ} \mathrm{S}$ to $10^{\circ} \mathrm{N}$ and from 0.50 to 0.06 bar.

slight northward displacement of the equatorial temperature minimum for $\mu=0.2,0.4$, and 0.6 at $20 \mu \mathrm{m}$ (Figure 1). Thus temperatures near the 0.06 -bar level might be $\sim 1 \mathrm{~K}$ lower as a result of the $\sim 50$-day passage of the ring shadow from north to south at latitudes from $2^{\circ}$ to $5^{\circ} \mathrm{N}$.

Figure 12 displays the vertical temperature profiles for cases 1,2 , and 3 for both the $13.5^{\circ}-16.5^{\circ} \mathrm{S}$ and the $1.5^{\circ} \mathrm{N}$ to $1.5^{\circ} \mathrm{S}$ regions. With these we compare the radio occultation results of Kliore et al. [this issue], the semiempirical equilibrium model of Tokunaga and Cess [1977], and one of the equilibrium models of J. F. Appleby and J. Hogan (unpublished manuscript, 1980). The structural details of the Kliore et al. model are only crudely approximated by the constant lapse rate adopted in cases 1 and 3. The agreement in the 125- to 60-mbar region between this result and the results derived from the IRR data has been optimized by means of adjustment of the bulk composition.

Assumption of a steeper overlying inverted lapse rate, as is consistent with the model of Tokunaga and Cess, substantially changes only the 63-mbar nodal point in our retrieved temperatures and does not increase the residuals of the model fit to the data in any systematic way. It is therefore quite possible to achieve a consistency between our derived thermal structure models and either of the equilibrium models. Appleby and Hogan have shown, in fact, that it is possible to change the stratospheric temperature structure substantially with appropriate changes in the vertical distribution of absorbing aerosols. Furthermore, as was mentioned earlier, the equilibrium models are consistent with the assumption in case 3 models that the atmosphere is in convective equilibrium near the 501-mbar level.

Models fitting the microwave spectrum of Saturn have been presented by Klein et al. [1978]. Unlike the case for Jupiter, no high-resolution data are available in the spectral region around several strong inversion lines of $\mathrm{NH}_{3}$. Their results are accordingly constrained only to a family of models in which the mixing ratio of ammonia in the deep atmosphere is parameterized against a given temperature structure (essentially, a given adiabat). These models thus provide no independent verification of the temperatures retrieved here. However, the models we present here can be used with the microwave data to constrain the abundance of $\mathrm{NH}_{3}$ and $\mathrm{H}_{2} \mathrm{O}$ in the deep atmosphere, as is discussed by Klein et al. [1978].

\section{SPECTRA}

The models presented in the preceding section have been used to generate low-resolution spectra in the $25-$ to $800-\mathrm{cm}^{-1}$ region. These are summarized in Figure 13. For convenience, in comparison with many earth-based observations the hemispherically averaged brightness temperatures (equivalent whole disk) have been plotted. This figure also shows a schematic plot of the spectral response of the 20 - and $45-\mu \mathrm{m}$ filters. The spectra are dominated by the collision-induced dipole opacity of $\mathrm{H}_{2}$. Absorption peaks for the $S(0)$ and the $S(1)$ rotational lines are near 375 and $600 \mathrm{~cm}^{-1}$, respectively. These spectral regions appear rather isothermal, because radiation emerging from them is originating to a large extent from the rather isothermal regions of the atmosphere near 100 mbar. At lower frequencies the translational absorption band of $\mathbf{H}_{2}$ is apparent, and it becomes increasingly transparent at frequencies below $100 \mathrm{~cm}^{-1}$. The spectra of different model cases are not very different from one another and are denoted by the separation between the solid curves representing each region in Figure 13.

The general agreement of earth-based observations with the spectra computed from the models is good. The feature near $830 \mathrm{~cm}^{-1}$ in the Gillett and Forrest [1974] spectrum is due to the $\nu_{9}$ fundamental band of ethane $\left(\mathrm{C}_{2} \mathrm{H}_{6}\right)$, whose opacity was not included in the calculation of the spectra shown. Between 400 and $600 \mathrm{~cm}^{-1}$ most of the observations were made with some degree of spatial resolution of the disk, and so a comparison with the hemispherically averaged spectrum may not be wholely appropriate. The Erickson et al. [1978] spectrum, shown somewhat schematically at a lower resolution than observed, includes flux from the entire disk and from the ring system. The observations by R. F. Loewenstein et al. (unpublished manuscript, 1980) and Hildebrand et al. [1980] are displayed also, as they were made during times when the ring system subtended an angle of $1^{\circ}$ or less as viewed from the earth, so that their flux contribution relative to that of the planetary disk is considered to be negligible.

The earth-based observations are largely in agreement with our predicted spectra. A notable exception is the 40 - to 100 $\mathrm{cm}^{-1}$ spectral region, where broad-band-pass-filtered radiometry displays a substantial drop in brightness compared with that in our models. If we choose to interpret this as a real feature, then absorption by $\mathrm{NH}_{3}$ or $\mathrm{H}_{2} \mathrm{O}$ vapor is extremely unlikely if we assume that these constituents are in saturation equilibrium at the ambient temperatures. Other possible constituents (e.g., $\mathrm{PH}_{3}, \mathrm{CO}$ ) must also meet the constraints imposed by their influence on other spectral regions. Ice candidates such as $\mathrm{NH}_{3}$ [Taylor, 1973] or even $\mathrm{H}_{2} \mathrm{O}$ [Irvine and Pollack, 1968] do not have strong absorption in this region and are therefore unlikely to succeed in explaining the observed drop in brightness. Alternatively, it is possible that this feature is an artifact of the earth-based calibration, providing that there is a corresponding spectral feature in the atmosphere of Mars (the primary calibration source for both Loe- 


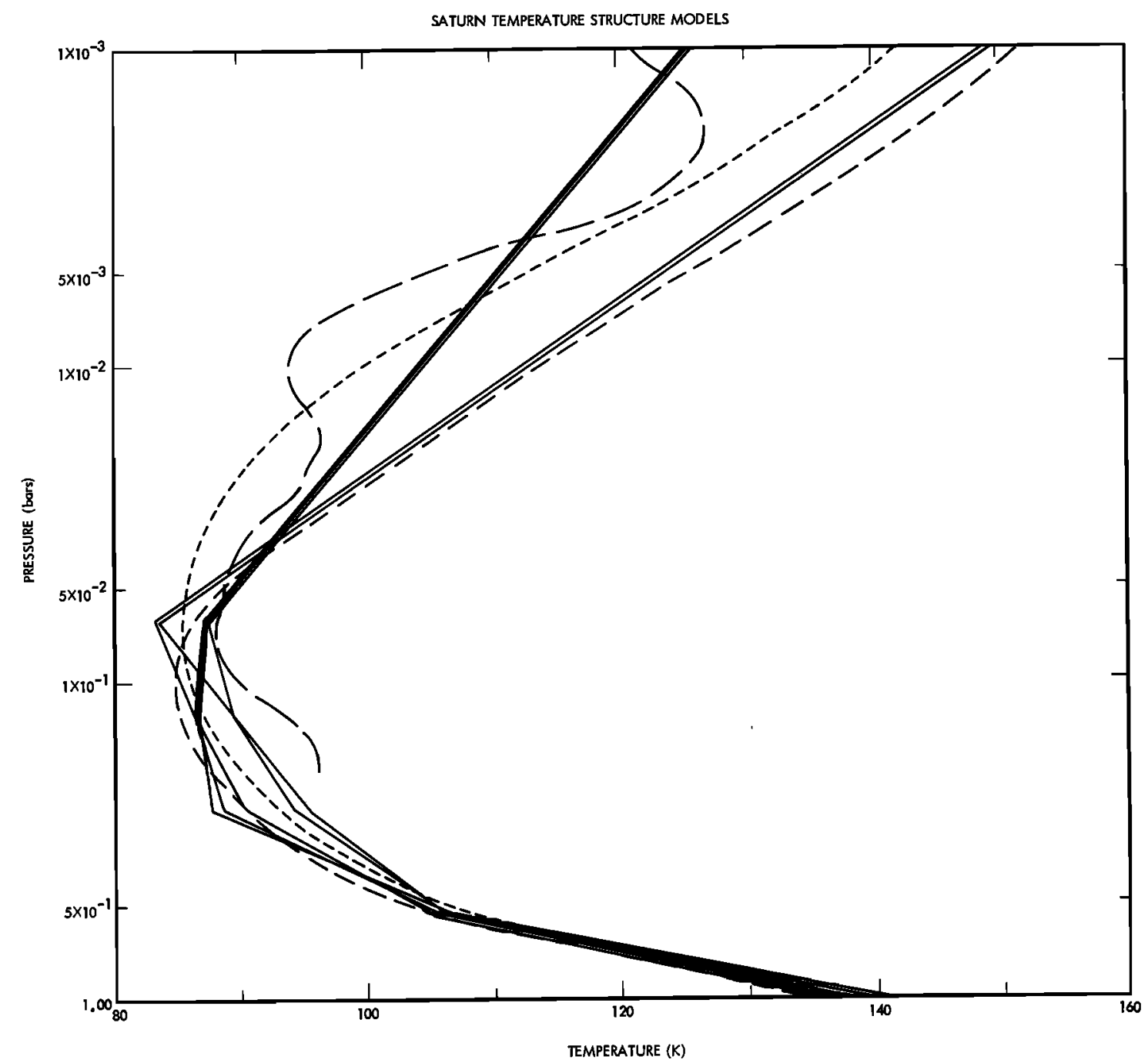

Fig. 12. Comparison of temperature structures here with some other models. Solid lines represent the temperature structures shown in Figure 7 (cases 1 and 2) and Figure 8 (cases 1, 2, and 3). At pressures less than $3 \times 10^{-2}$ bar, cases 1 and 3 (three lines) lie to the left of case 2 (two lines). At pressures between $10^{-1}$ and $5 \times 10^{-1}$ bar the profiles from Figure 7 (two lines) lie to the right of those from Figure 8 (three lines). Long dashes represent the temperature structure derived by Kliore et al. [this issue] from inversion of Pioneer 11 radio occultation egress data. Intermediate dashes represent the global model of Tokunaga and Cess [1977]. Short dashes represent the model of J. F. Appleby and J. Hogan (unpublished manuscript, 1980) in which $15 \%$ of the incident sunlight is absorbed uniformly in the stratosphere.

wenstein et al. and Hildebrand et al.). We suggest that higherresolution spectral observations of this region would at least verify the presence of the feature as well as determine whether distinct absorption features belonging to a gas (as opposed to the relatively broader features of an ice or liquid) are detectable.

\section{TOTAL INFRARED BRIGHTNESS}

The total infrared flux from each of the latitude regions observed was computed by using model spectra such as those shown in Figure 13. The model spectrum was integrated from 0 to $750 \mathrm{~cm}^{-1}$, and the observed disk spectrum of Gillett and Forrest [1974] was used at higher frequencies. The results are shown in Figure 14. We see immediately that a strong symmetry exists about the equator and relatively constant values appear between $7.5^{\circ} \mathrm{S}$ and $22.5^{\circ} \mathrm{S}$ latitude. Poleward of $22.5^{\circ} \mathrm{S}$ the data imply a total flux output less than that in the $7.5^{\circ}$ $22.5^{\circ} \mathrm{S}$ region. However, we note that the data are substantially fewer in number for these regions (as in the case for latitudes greater than $15^{\circ} \mathrm{N}$ ) than for latitude bins in the $7.5^{\circ}-$ $22.5^{\circ} \mathrm{S}$ region.

The effective temperatures quoted by Ingersoll et al. [1980] are some 1.5-2.0 K cooler than those shown in Figure $14 \mathrm{ow}-$ ing to an incorrect treatment of the opacities in the $100-\mathrm{cm}^{-1}$ region, which produced an underestimate of the flux. That treatment was, however, more consistent with the spectrum measurements of R. F. Loewenstein et al. (unpublished manuscript, 1980) and may be closer to the true value of the total output if the earth-based measurements are correct. However, for the sake of consistency we will use values which are derived from the theoretical spectra, dependent mostly on the $\mathbf{H}_{2}$ dipole opacity as shown in Figure 13. If we make the extremely simplifying assumptions of symmetry about the equator and a constant flux poleward of $7.5^{\circ} \mathrm{S}$, then the values for total thermal emission shown in Figure 14, integrated over the sphere, imply an effective planetary temperature

$$
T_{\text {eff }}=96.5 \pm 2.5 \mathrm{~K}
$$




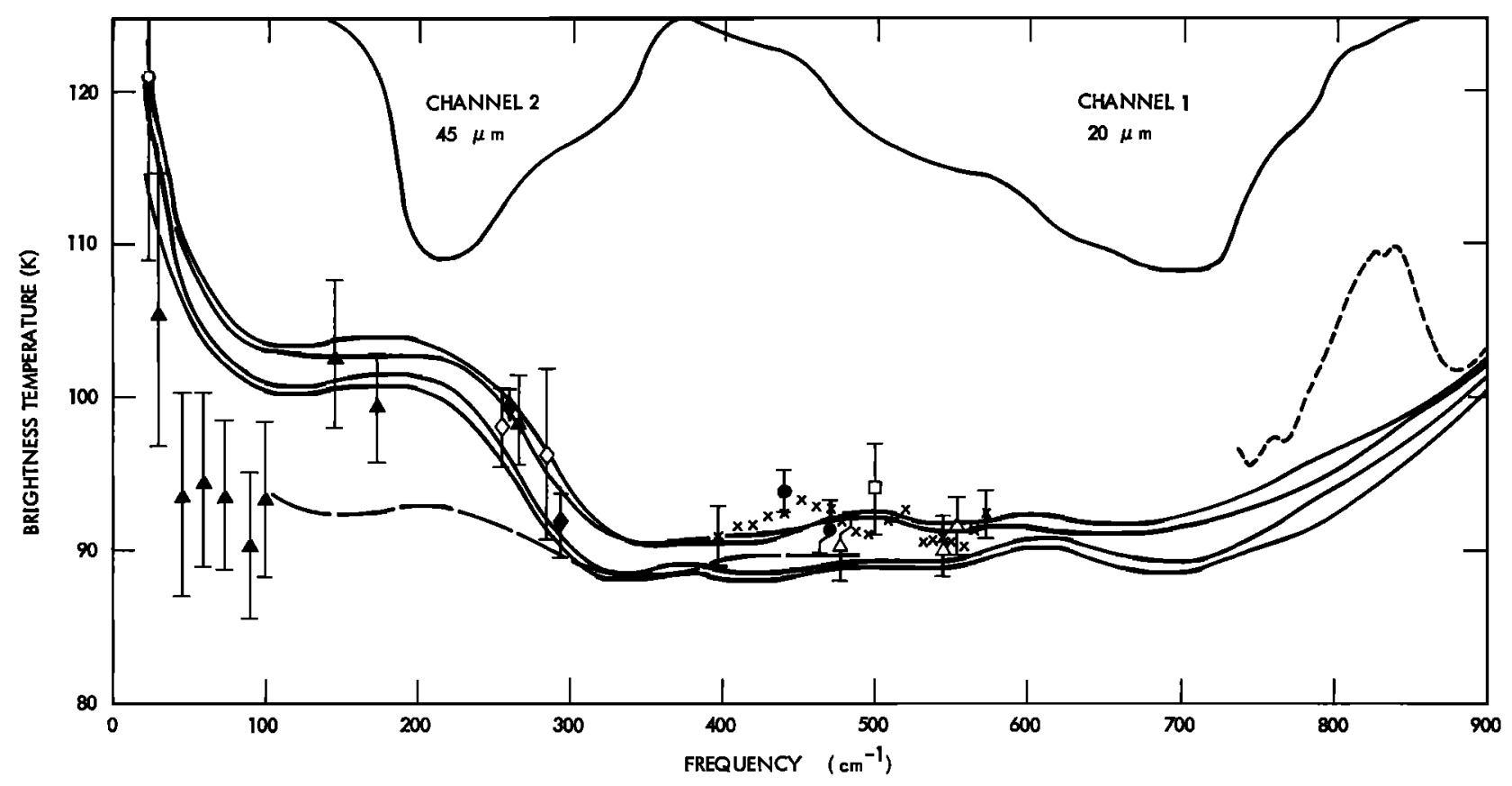

Fig. 13. Comparison of the spectra of models derived in this paper with earth-based observations. ' $T$ te upper pair of solid curves represent bounds of all spectra computed for the warm $13.5^{\circ}-16.5^{\circ} \mathrm{S}$ latitude region. The lower pair of solid curves are bounds for the cool $1.5^{\circ} \mathrm{S}$ to $1.5^{\circ} \mathrm{N}$ latitude region. The effective resolution element of the calculated spectrum is $25 \mathrm{~cm}^{-1}$. The opacity of $\mathrm{NH}_{3}$ vapor is included, calculated by using the band model parameters of Gille and Lee [1969]. The opacity of $\mathrm{C}_{2} \mathrm{H}_{6}$ (at $825 \mathrm{~cm}^{-1}$ ) is not included. Curves at the top are the relative responses of the two channels. Long dashes and short dashes are somewhat schematic representations of the spectra of Erickson et al. [1978] and of Gillett and Forrest [1974], respectively. Crosses represent the spectra of Tokunaga et al. [1977] with representative error bars at the high- and low-frequency extent of the data. Filtered photometric observations are represented at appropriate effective frequencies by solid circles [Rieke, 1975], open diamonds [Nolt et al., 1977], solid diamonds [Nolt et al., 1978], open triangles [Knacke et al., 1975], open square [Morrison, 1974], solid triangles (R. F. Loewenstein et al., unpublished manuscript, 1980), and open circle [Hildebrand et al., 1980]. An additional open circle lies off the scale at $139 \mathrm{~K}, 12.5 \mathrm{~cm}^{-1}$.

The uncertainty quoted largely reflects the calibration uncertainty. The uncertainties involved in the model extrapolation to long wavelengths and to latitudes south of $31.5^{\circ} \mathrm{S}$ and north of $10.5^{\circ} \mathrm{N}$ are not included in this value, as we do not know of a cogent way to quantify them explicitly.

\section{GLOBAL ENERGY BUdGeT AND INTERIOR MODELS}

Saturn's total emitted power per unit area is about $4.9 \pm 0.5$ $\mathrm{W} \mathrm{m} \mathrm{m}^{-2}$ according to (3). This value is about $0.4 \mathrm{~W} \mathrm{~m}^{-2}$ higher than that given in our preliminary report. In addition, there is some evidence [Tomasko et al., this issue] that the phase integral of Saturn is about 1.50 instead of 1.25 , as observed for Jupiter. This raises the estimate of the Bond albedo $A$ from $0.45 \pm 0.15$ [Erickson et al., 1978] to $0.54 \pm 0.15$. The average internal heat flux is then $3.2 \pm 1.0 \mathrm{~W} \mathrm{~m}^{-2}$, and the ratio of total emitted power to sunlight absorbed is $2.8 \pm 0.9$. The phase integral and Bond albedo are still preliminary estimates, however, and the emitted flux was computed with no information poleward of about $40^{\circ} \mathrm{S}$. Thus inferences about the internal energy source are still uncertain and subject to change.

Nevertheless, it is clear from models of the cooling history and interior [Pollack et al., 1977; Stevenson, 1980] that $3.2 \pm$ $1.0 \mathrm{~W} \mathrm{~m}^{-2}$ is a large internal heat flux for Saturn. For Jupiter the Pioneer 10 and 11 IRR gave an effective temperature of $125 \pm 3 \mathrm{~K}$ [Ingersoll et al., 1976]. The photopolarimeter gave a phase integral of about 1.25 and a Bond albedo of 0.35 [Tomasko et al., 1978]. When combined, these imply an internal heat flux of $5.6 \mathrm{~W} \mathrm{~m}^{-2}$, a value that agrees with models of Jupiter's cooling history starting with gravitational collapse 4.5 b.y. ago [Graboske et al., 1975].
However, the new value of Saturn's internal heat flux is probably too large to be explained by simple cooling and contraction [Pollack et al., 1977; Stevenson, 1980]. An additional energy source, precipitation of 'telium at the top of a metallic hydrogen liquid interior, could supply some of the extra energy [Kieffer, 1967; Stevenson and Salpeter, 1977], but even this energy supply is limited. Stevenson [1980] estimates that depletion from an initial $25 \%$ helium by mass $\left(\alpha_{\mathrm{H}_{2}} \approx 0.86\right)$ to a present value of $15 \%\left(\alpha_{\mathrm{H}_{2}} \approx 0.92\right)$ in the outer molecular layer could account for a total internal heat flux at present of $2.4 \mathrm{~W}$ $\mathrm{m}^{-2}$, our preliminary published estimate [Ingersoll et al.,

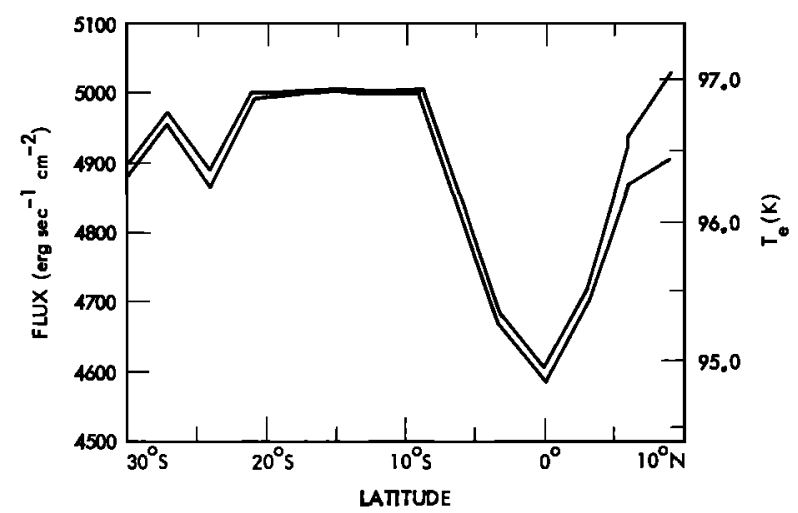

Fig. 14. Total outgoing infrared flux (or local effective temperature) plotted as a function of latitude. Values are derived from integration of the appropriate spectra along with consideration of earthbased observations [Gillett and Forrest, 1974] for frequencies greater than $750 \mathrm{~cm}^{-1}$. 
1980]. Precipitation of additional helium does not result in a substantially higher heat at present. Thus we might expect depletion of helium in the atmosphere of Saturn, though perhaps not on Jupiter, to explain the excess internal heat flux. Such a difference between the two planets is consistent with interior models [Stevenson and Salpeter, 1977].

Direct estimates of the atmospheric hydrogen to helium ratio are clearly important. The atmosphere is likely to reflect the bulk composition of the entire molecular envelope, because convection provides rapid mixing. The hydrogen to helium ratio for the planet as a whole is likely to be no greater than the solar composition value, for which $\alpha_{\mathrm{H}_{2}}=0.88$ according to one estimate [Cameron, 1974]. The uncertainty in this solar value is difficult to establish. But neither our estimate for Saturn's atmosphere, $\alpha_{\mathrm{H}_{2}}=0.90 \pm 0.03$, nor Gautier et al.'s [1980] estimate for Jupiter's atmosphere, $\alpha_{\mathrm{H}_{2}}=0.897 \pm 0.024$, is significantly different from the solar value, though both are high. It is possible that helium depletion has taken place on both planets, but the evidence is inconclusive. Thus it appears that our measurements are not quite accurate enough to resolve these important questions about the histories of the giant planets.

Acknowledgments. The Pioneer project staff provided reliable support during all phases of the mission. J. D. Bennett, M. Schroeder, B. Schupler, and J. C. Ingersoll developed computer codes for analyzing the data. G. Munch, G. Neugebauer, and S. C. Chase presided at the creation of this simple but reliable instrument. R. A. Hanel provided a helpful review of the manuscript. We thank them all.

The Editor thanks R. A. Hanel for his assistance in evaluating this paper.

\section{REFERENCES}

Caldwell, J., The atmosphere of Saturn: An infrared perspective, Icarus, 22, 57-65, 1977.

Cameron, A. G. W., Abundances of the elements in the solar system, Space Sci. Rev., 15, 121-146, 1974.

Chahine, M. T., An analytical transformation for remote sensing of clear-column atmospheric temperature profiles, J. Atmos. Sci., 32, 1946-1952, 1975.

Conrath, B. J., Vertical resolution of temperature profiles obtained from remote radiation measurements, J. Atmos. Sci, 29, 1262-1271, 1972.

Erickson, E. F., D. Goorvitch, J. Simpson, and D. W. Strecker, Farinfrared spectrophotometry of Jupiter and Saturn, Icarus, 35, 61$73,1978$.

Froidevaux, L., and A. P. Ingersoll, Temperatures and optical depths of Saturn's rings and a brightness temperature for Titan, $J$ Geophys. Res., this issue.

Gautier, D., A. Chedin, B. Conrath, R. Hanel, V. Kunde, G. Lindal, and $G$. Orton, The helium abundance of Jupiter, submitted to $J$. Geophys. Res., 1980.

Gille, J., and T.-H. Lee, The spectrum and transmission of ammonia under Jovian conditions, J. Atmos. Sci., 26, 932-940, 1969.

Gillett, F. C., and W. J. Forrest, The 7.5 to 13.5 micron spectrum of Saturn, Astrophys. J., 187, L37-L39, 1974.

Graboske, H. C., J. B. Pollack, A. S. Grossman, and R. J. Olness, The structure and evolution of Jupiter: The fluid contraction stage, Astrophys. J., 199, 265-281, 1975.

Hildebrand, R., J. Keene, and S. Whitcomb, Brightness temperatures of Saturn's disk and rings at 400 and $800 \mu \mathrm{m}$, Science, in press, 1980 .

Ingersoll, A. P., G. Münch, G. Neugebauer, and G. S. Orton, Results of the infrared radiometer experiment on Pioneers 10 and 11 , in $\mathrm{Ju}$ piter, edited by T. Gehrels, pp. 197-205, University of Arizona Press, Tucson, 1976.

Ingersoll, A. P., G. S. Orton, G. Münch, G. Neugebauer, and S. C. Chase, Pioneer Saturn infrared radiometer: Preliminary results, Science, 207, 439-443, 1980.

Irvine, W. M., and J. B. Pollack, Infrared optical properties of water and ice spheres, Icarus, 8, 324-360, 1968.

Kieffer, H. H., Calculated physical properties of planets in relation to composition and gravitational layering, J. Geophys. Res., 72, 31793197, 1967

Klein, M. J., M. A. Janssen, S. Gulkis, and E. T. Olsen, Saturn's microwave spectrum: Implications for the atmosphere and the rings, NASA Conf. Publ., 2068, 195-216, 1978.

Kliore, A. J., G. F. Lindal, I. R. Patel, D. N. Sweetnam, H. B. Hotz, and T. R. McDonough, Vertical structure of the ionosphere and the upper neutral atmosphere of Saturn from the Pioneer radio occultation, Science, 207, 446-449, 1980.

Kliore, A. J., I. R. Patel, G. F. Lindal, D. N. Sweetnam, H. B. Hotz, J. H. Waite, Jr., and T. R. McDonough, Structure of the ionosphere and atmosphere of Saturn from Pioneer 11 Satum radio occultation, J. Geophys. Res., this issue.

Knacke, R. F., T. Owen, and R. R. Joyce, Infrared observations of the surface and atmosphere of Titan, Icarus, 24, 460-464, 1975.

Morrison, D., Infrared radiometry of the rings of Saturn, Icarus, 22, 57-65, 1974

Nolt, I. G., W. M. Sinton, L. J. Caroff, E. F. Erickson, D. W. Strecker, and J. V. Radostitz, The brightness temperature of Saturn and its rings at 39 microns, Icarus, 30, 747-759, 1977.

Nolt, I. C., A. Tokunaga, F. C. Gillett, and J. Caldwell, The $22.7 \mathrm{mi}-$ cron brightness of Saturn's rings versus declination of the sun, $A s$ trophys. J. 219, L63-L66, 1978.

Orton, G. S., The thermal structure of Jupiter, I, Implications of Pioneer 10 infrared radiometer data, Icarus, 26, 125-141, 1975.

Orton, G. S., Recovery of the mean Jovian temperature structure from inversion of spectrally resolved thermal radiance data, Icarus, $32,41-57,1977$.

Orton, G. S., and A. P. Ingersoll, Pioneer 10 and 11 and ground-based infrared data on Jupiter: The thermal structure and $\mathrm{He}-\mathrm{H}_{2}$ ratio, in Jupiter, edited by T. Gehrels, pp. 207-215, University of Arizona Press, Tucson, 1976.

Pollack, J. B., A. S. Grossman, R. Moore, and H. C. Graboske, A calculation of Saturn's gravitational contraction history, Icarus, 30, 111-128, 1977.

Rieke, G. H., The thermal radiation of Saturn and its rings, Icarus, 26, 37-44, 1975 .

Stevenson, D. J., Saturn's luminosity and magnetism, Science, 208, 746-748, 1980.

Stevenson, D. J., and E. E. Salpeter, The dynamics and helium distribution in hydrogen-helium fluid planets, Astrophys. J. Suppl. Ser., 35, 239-261, 1977

Taylor, F. W., Preliminary data on the optical properties of solid ammonia and scattering parameters for ammonia cloud particles, $J$. Atmos. Sci., 30, 677-683, 1973.

Tokunaga, A., and R. D. Cess, A model for the temperature inversion within the atmosphere of Saturn, Icarus, 32, 321-327, 1977.

Tokunaga, A., R. F. Knacke, and T. Owen, 17-25 micron spectra of Jupiter and Saturn, Astrophys. J., 213, 569-574, 1977.

Tomasko, M. G., R. A. West, and N. D. Castillo, Photometry and polarimetry of Jupiter at large phase angles, I, Analysis of imaging data of a prominent belt and a zone from Pioneer 10, Icarus, 33, 558-592, 1978.

Tomasko, M. G., R. S. McMillan, L. R. Doose, N. D. Castillo, and J. P. Dilley, Photometry of Saturn at large phase angles, J. Geophys. Res., this issue.

(Received May 5, 1980;

revised June 2, 1980;

accepted June 3, 1980.) 